\title{
Nuevos tratamientos médicos para el cáncer medular de tiroides
}

\author{
New therapies for medullary thyroid carcinoma
}

\author{
Román-González $A^{1,2}$, Mejía $S^{3}$, Zapata $M^{3,4}$.
}

\author{
${ }^{1}$ Médico Internista Endocrinólogo. Servicio de Endocrinología \\ y Metabolismo, Hospital Universitario San Vicente Fundación. \\ Medellín, Colombia. \\ 2Profesor de endocrinología, Sección de Endocrinología y Diabetes, \\ Departamento de Medicina Interna, Universidad de Antioquia. \\ Medellín, Colombia. \\ ${ }^{3}$ Médico Internista Oncologo Servicio de Oncología, Hospital \\ Universitario San Vicente Fundación. Medellín, Colombia. \\ 4. Profesor Oncología, Universidad de Antioquia \\ Autor de correspondencia: Alejandro Román-González \\ Correo electrónico: alejoroman@gmail.com \\ Fecha de recepción: 29/04/2019 \\ Fecha de aceptación: 30/10/2019
}

\section{Resumen}

Los pacientes con cáncer medular de tiroides avanzado tienen pocas opciones de tratamiento; las únicas terapias aceptadas a la fecha son el vandetanib y el cabozantinib. Sin embargo, al ser inhibidores multiquinasa tienen efectos adversos importantes, que pueden limitar su uso clínico. Con el vandetanib la prolongación del QT puede producir arritmias y requiere un uso cauteloso de medicamentos concomitantes. Con el cabozantinib, aparte de la hipertensión y el síndrome mano-pie, pueden aparecer fistulas y perforación gastrointestinal con riesgo de sangrado.

Las alteraciones genéticas del proto-oncogén RET no solo están implicadas en el cáncer medular de tiroides, sino en otros tipos de cáncer, como el de pulmón. La inhibición específica del RET con los medicamentos LOXO-292 y BLU-677 es segura y no afecta otras tirosina quinasa. Por ende, su uso clínico parece ser prometedor, dado que tienen una buena respuesta clínica; además, tienen efectos adversos menos frecuentes y graves que los inhibidores de tirosina quinasa actuales.

Palabras clave: cáncer medular de tiroides, proteínas proto-oncogénicas c-ret, LOXO-292, BLU-667, cabozantinib, vandetanib.

\begin{abstract}
Patients with advanced medullar thyroid carcinoma have limited options. Current approved therapies for this cancer are vandetanib and cabozantinib. However, the non-specific inhibition produces side effects limiting its use in clinical practice. For example, vandetanib may prolongate QT and in rare cases trigger ventricular arrhythmias. Cabozantinib may be associated with hypertension, hand-and-foot syndrome, fistula and gastrointestinal perforation with bleeding.

RET alterations are associated with medullary thyroid carcinoma as well as other types of cancer such as lung cancer. Specific inhibition of this tyrosine kinase by LOXO-292 y BLU-677 is safe and limited only to RET, without inhibition of other tyrosine kinases. Therefore, its clinical use is promising given its excellent clinical responses and infrequent side effects compared to available tyrosine kinase inhibitors.

Keywords: medullary thyroid carcinoma, c-ret proto-oncogene proteins, LOXO-292, BLU-667, cabozantinib, vandetanib.
\end{abstract}

\section{Introducción}

El cáncer medular de tiroides $(\mathrm{CMT})^{(1)}$ es un tumor neuroendocrino que se deriva de las células parafoliculares o células $\mathrm{C}$ de la tiroides, las cuales se encargan de la producción de calcitonina ${ }^{(2)}$; y corresponde al $1 \%$-2 \% de las neoplasias tiroideas ${ }^{(3)}$. Sin embargo, en nuestro medio son pocos los datos conocidos de esta enfermedad, dado que la totalidad de los estudios son dedicados a la epidemiología del cáncer de tiroides.

Por ejemplo, en Manizales se hizo un seguimiento a 544 pacientes con cáncer de tiroides, de los cuales 1,3\% (n = 7) correspondían a CMT. La mayoría de los casos fueron esporádicos; se presentaron dos casos de cáncer medular familiar y uno de neoplasia endocrina múltiple tipo 2B (MEN 2B). La supervivencia a 5 años fue de $70 \%{ }^{(4)}$. En Popayán se analizaron pacientes con diagnóstico de cáncer de tiroides entre 2004 y 2013. De los 205 pacientes, solo 2 de ellos (0.975 \%) correspondían a CMT, ambas mujeres ${ }^{(5)}$. 
En Bogotá se estudiaron 501 pacientes con cáncer de tiroides entre 1997 y 2012, de los cuales solo 13 (2,6 \%) presentaban $\mathrm{CMT}^{(6)}$. En Bucaramanga entre 2001 y 2013 se encontraron 268 pacientes con cáncer de tiroides, de los cuales 5 tenían $\mathrm{CMT}^{(7)}$. El estudio multicéntrico más grande en Colombia evaluó 1096 pacientes con cáncer de tiroides, en el cual se encontraron 19 casos de CMT $(1,7 \%)^{(8)}$.

La mayoría del CMT es esporádico; es decir, no tiene una mutación por línea germinal. Sin embargo, hasta el 25 \% de los pacientes pueden tener como causa del CMT una neoplasia endocrina múltiple tipo 2 por mutaciones en el proto-oncogén $\operatorname{RET}^{(9)}$. La mayoría de los pacientes con esta enfermedad se benefician del manejo quirúrgico; y cuando el tumor es localizado, existe una oportunidad de lograr la curación del paciente. No obstante, la mayoría de los pacientes se diagnostican en estadios tardíos o con enfermedad avanzada e irresecable. En estos casos, el uso de inhibidores de tirosina quinasa puede tener utilidad clínica ${ }^{(10)}$, dado que la mayoría de estos tienen una inhibición no específica; pero los efectos adversos pueden limitar estas terapias.

Adicionalmente, los pacientes pueden progresar a las terapias establecidas. Es por ello por lo que se necesitan medicamentos más seguros y potentes en cáncer medular de tiroides. Enfocarse en el RET, como un blanco de tratamiento, emerge como una opción terapéutica prometedora, teóricamente con diferente perfil de seguridad. En esta revisión se presenta la evidencia en construcción de los inhibidores de RET LOXO292 y BLU-667 para cáncer medular de tiroides.

\section{Proto-oncogén RET}

RET, del inglés re-arranged during transfection, es un proto-oncogén que codifica una proteína tirosina quinasa ${ }^{(11)}$. Está localizado en el cromosoma 10q11.2 y está involucrado directamente en la patogénesis del carcinoma medular de tiroides $^{(11)}$. Casi todos los pacientes con CMT hereditario (neoplasia endocrina múltiple tipo 2A y 2B) y $50 \%-60 \%$ de los casos esporádicos presentan mutaciones somáticas en el RET. Este gen fue descubierto por Takahashi en $1985^{(12)}$ y está expresado en células derivadas de la cresta neural. En la Figura 1 se presenta un esquema representativo de este gen.

Las mutaciones en el proto-oncogen RET producen una activación constitutiva de la función quinasa de este receptor; por consiguiente, las vías de señalización de este receptor se mantienen activas y generan una proliferación de las células $\mathrm{C}$ parafoliculares de la tiroides. La proteína RET tiene un dominio de unión al ligando extracelular, que está compuesto de una región tipo cadherina y un dominio altamente conservado rico en cisteína. La región intracelular está compuesta por dos dominios tirosina quinasa. La unión del ligando hace que el dominio rico en cisteína facilite la dimerización del receptor y produzca autofosforilación y activación de la tirosina quinasa y de las vías de señalización, que incluyen RAS/RAF/MAPK, PI3K/AKT y JAK/ STAT. El RET produce neoplasias por dos mecanismos principales: por mutaciones o por fusiones en $\operatorname{RET}^{(13)}$.

Figura 1. Esquema representativo del proto-oncogén RET.

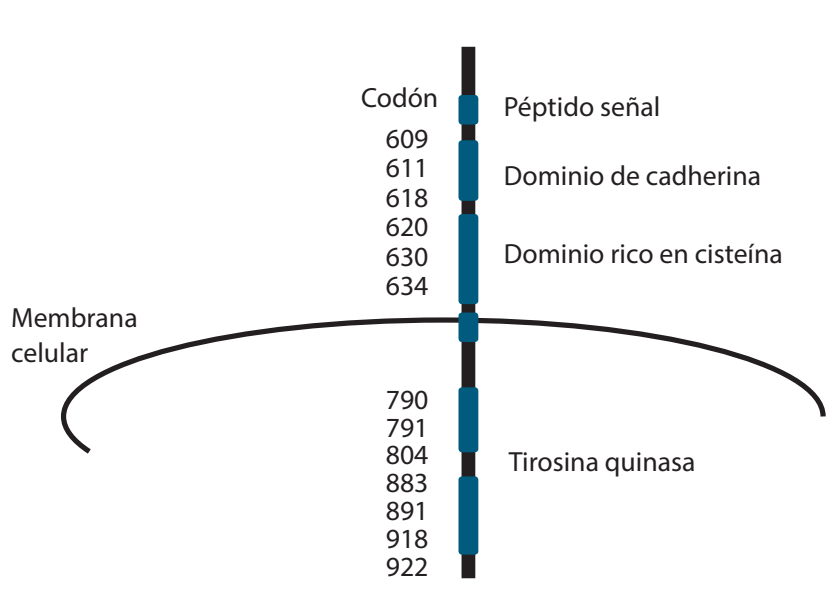

Las mutaciones germinales en este proto-oncogén producen el síndrome de neoplasia endocrina múltiple tipo 2A (MEN 2A) y la neoplasia endocrina múltiple tipo 2B (MEN 2B). El MEN 2A incluye CMT, hiperparatiroidismo y feocromocitoma. Se divide en: MEN 2A clásico, MEN 2A con liquen amiloidosis cutáneo, MEN 2A con enfermedad de Hirschsprung y CMT familiar.

Las manifestaciones clínicas dependen de los órganos comprometidos y esto, a su vez, es dependiente de la mutación específica. La penetrancia del CMT es del 100 \%, pero es variable en las otras manifestaciones. En términos generales, la penetrancia de feocromocitoma es del $50 \%$ y del hiperparatiroidismo es del $20 \%$. Los porcentajes pueden variar según la mutación que tenga el paciente y la edad de seguimiento. A mayor seguimiento, mayor probabilidad de que se presente una manifestación clínica. En la Tabla 1 se presentan las diferentes manifestaciones de acuerdo con el tipo de mutación.

El MEN 2B se asocia con CMT, feocromocitoma, ausencia de hiperparatiroidismo y un fenotipo característico con hábito marfanoide ${ }^{(14)}$. Es una enfermedad autosómica dominante y el CMT de este es más agresivo en comparación con el CMT del MEN 2A. Los tumores se presentan en edad temprana y usualmente la cirugía no es curativa. Otras manifestaciones del MEN 2B son neuromas en mucosas, en particular en la lengua y los labios, ganglioneuromas, deformidades esqueléticas (lordosis y cifoescoliosis), constipación y megacolon.

Los pacientes con CMT aparentemente esporádico tienen mutaciones en el RET por línea germinal hasta el $7 \%$ de los casos y de forma somática en el $50 \%-60 \%{ }^{(15)}$. El RET está implicado en la patogénesis de varios tipos de cáncer, como el papilar de tiroides, el carcinoma no microcítico de pulmón y el cáncer medular de tiroides ${ }^{(16)}$. Otros tipos de cáncer asociados con alteraciones en el RET, en particular fusiones del RET, son el cán- 
cer de colon, mama, ovarios, carcinomas de glándulas salivales, leucemia mieloide crónica y tumores miofibroblásticos inflamatorios $^{(11,13,17-20)}$. También se han descrito fusiones en el RET en baja frecuencia en cáncer de páncreas y tumores de Spitz ${ }^{(21)}$.

\section{Tratamiento estándar del cáncer medular de tiroides}

Los pacientes con CMT son diagnosticados mediante una biopsia de un nódulo tiroideo o en la patología final luego de una tiroidectomía. El tratamiento de esta enfermedad es mediante cirugía con tiroidectomía total ${ }^{(15)}$. La evaluación de un paciente con sospecha o con diagnóstico confirmado incluye una ecografía de tiroides con mapeo ganglionar, medición de calcitonina, antígeno carcinoembrionario (ACE), prueba genética para descartar mutaciones germinales en el RET y siempre se debe descartar feocromocitoma antes de la cirugía, idealmente mediante la medición de metanefrinas fraccionadas en plasma ${ }^{(15,22)}$.

También se debe evaluar la presencia de hiperparatiroidismo mediante la medición del calcio y la parathormona. En algunos casos, se puede omitir la evaluación del feocromocitoma e hiperparatiroidismo si el paciente no tiene mutaciones en el RET. Si el pacientes tiene elevación preoperatoria marcada de calcitonina $(>500 \mathrm{pg} / \mathrm{mL})$ se debe incluir una tomografía contrastada de cuello y tórax, resonancia de hígado y de esqueleto axial. Contrario a lo que ocurre con el carcinoma diferenciado de tiroides (papilar y folicular), las células del CMT no captan el yodo y, por consiguiente, esta terapia no tiene efectividad en esta enfermedad.

Tabla 1. Correlación entre las mutaciones y el fenotipo en el síndrome de neoplasia endocrina múltiple

\begin{tabular}{|c|c|c|c|c|c|c|c|}
\hline Exón & Codón/mutaciones & CMT F & MEN 2A & HSCR & CLA & HPT & MEN 2B \\
\hline 5 & G321R & + & - & - & - & - & - \\
\hline 8 & G533C & + & - & - & - & - & - \\
\hline 10 & $\begin{array}{c}\mathrm{R} 600 \mathrm{Q} \\
\mathrm{K} 603 \mathrm{E} \\
\mathrm{Y} 606 \mathrm{C} \\
\mathrm{C} 609 \mathrm{~F} / \mathrm{R} / \mathrm{G} / \mathrm{S} / \mathrm{Y} / \mathrm{W} \\
\mathrm{C} 611 \mathrm{R} / \mathrm{G} / \mathrm{F} / \mathrm{S} / \mathrm{W} / \mathrm{Y} \\
\mathrm{C} 618 \mathrm{R} / \mathrm{G} / \mathrm{F} / \mathrm{S} / \mathrm{Y} \\
\mathrm{C} 620 \mathrm{R} / \mathrm{G} / \mathrm{F} / \mathrm{S} / \mathrm{W} / \mathrm{Y}\end{array}$ & $\begin{array}{l}+ \\
+ \\
+ \\
+ \\
+ \\
+ \\
+\end{array}$ & $\begin{array}{l}+ \\
+ \\
+ \\
+\end{array}$ & $\begin{array}{l}+ \\
+ \\
+ \\
+\end{array}$ & & $\begin{array}{l}+ \\
+ \\
+ \\
+\end{array}$ & \\
\hline 11 & $\begin{array}{c}\mathrm{C630R/F} / \mathrm{S} / \mathrm{Y} \\
\mathrm{C634R/G/F/S} / \mathrm{W} / \mathrm{Y} \\
\mathrm{S} 649 \mathrm{~L} \\
\mathrm{~K} 666 \mathrm{E}\end{array}$ & $\begin{array}{l}+ \\
+ \\
+ \\
+\end{array}$ & $\begin{array}{l}+ \\
+ \\
+\end{array}$ & & + & $\begin{array}{l}+ \\
+\end{array}$ & \\
\hline 13 & $\begin{array}{l}\text { E768D } \\
\text { Q781R } \\
\text { L790F } \\
\text { Y791F } \\
\text { N777S }\end{array}$ & $\begin{array}{l}+ \\
+ \\
+ \\
+ \\
+\end{array}$ & + & & & & \\
\hline 14 & V804L/M & + & + & & + & + & \\
\hline 15 & $\begin{array}{l}\text { A883F } \\
\text { S891A }\end{array}$ & $\begin{array}{l}+ \\
+\end{array}$ & + & & & + & + \\
\hline 16 & $\begin{array}{l}\text { R912P } \\
\text { M918T }\end{array}$ & $\begin{array}{l}+ \\
+\end{array}$ & & & & & + \\
\hline
\end{tabular}

CMTF: carcinoma medular de tiroides familiar; MEN 2A: neoplasia endocrina múltiple tipo 2A; MEN 2B: neoplasia endocrina múltiple tipo 2B; HSCR: enfermedad de Hirschsprung; CLA: liquen amiloidosis cutáneo; HPT: hiperparatiroidismo primario.

Fuente: Elaboración propia 
Una vez la enfermedad está avanzada y se han agotado las alternativas de manejo, como radioterapia, radiocirugía, ablación con radiofrecuencia o embolización de las metástasis, quedan pocas opciones. En estos casos, las agencias regulatorias aprobaron el uso de inhibidores multiquinasa, como el cabozantinib y el vandetanib ${ }^{(9,23-29)}$. Estas terapias tienen una toxicidad importante que debe ser manejada apropiadamente; en el tiempo, algunos pacientes progresan a estos tratamientos o tienen contraindicaciones para su uso apropiado.

En nuestro medio y a la fecha de publicación de este artículo no está disponible ninguna de estas terapias; y el vandetanib se debe tramitar ante el Instituto Nacional de Vigilancia de Medicamentos y Alimentos (INVIMA) para autorizar su importación como un medicamento vital no disponible. Parte de la limitación para el uso del vandetanib es la prolongación del QTc, lo que contraindicaría esta terapia. Se espera que en los próximos años tengamos disponible del cabozantinib en Colombia.

Dado que la base molecular de esta enfermedad es la alteración en el RET, la existencia de inhibidores de este gen puede ser una opción más atractiva, con un uso más preciso y teóricamente con menos efectos adversos ${ }^{(13,16,17)}$.

\section{BLU-667}

BLU-667 es un inhibidor potente y selectivo del RET ${ }^{(17)}$. La media de concentración inhibitoria máxima (IC50) para la mutación RET M918T es de 0,4 nm. En cambio, respeta VEGFR con un IC50 de $35 \mathrm{~nm}$. Esto indica que es un inhibidor de RET altamente selectivo. En estudios in vitro, el BLU-667 ha demostrado una potencia 10 veces mayor que los inhibidores multiquinasa contra variantes del RET. Inhibe el crecimiento en modelos xenográficos de cáncer de tiroides y de pulmón. En pruebas en humanos, el BLU-667 ha demostrado su capacidad para inhibir la señalización por el RET y además genera respuestas en cáncer de tiroides y en cáncer pulmonar, al parecer, con una toxicidad mínima.

El estudio ARROW fase I presentó sus resultados preliminares en el $88^{\text {th }}$ Annual Meeting of the American Thyroid Association. Es el primer estudio en humanos con este medicamento; y su objetivo era probar el concepto de inhibir el RET y establecer la dosis máxima tolerada. En este primer reporte se incluyeron 69 pacientes $^{(30)}$. El $94 \%$ de estos tenían enfermedad metastásica y el $74 \%$ habían recibido algún inhibidor multiquinasa previamente. Se incluyeron 37 pacientes con carcinoma medular de tiroides, 5 pacientes con carcinoma papilar de tiroides con fusión en el RET, 23 pacientes con carcinoma no microcítico de pulmón, 1 paciente con colangiocarcinoma con fusión en el RET y 1 paciente con paraganglioma retroperitoneal y dos tumores sólidos sin mutaciones en el RET.

Además, comprendía una segunda parte de extensión, que incluyó pacientes con cáncer medular de tiroides, cáncer no microcítico de pulmón y otros tumores sólidos. La dosis usada fue 400 mg vía oral cada día. El 90 \% de los pacientes con cáncer de tiroides con alteración en el RET tuvieron una disminución en el tamaño del tumor. Se encontró 1 respuesta completa, $46 \%$ de respuesta parcial y $51 \%$ de enfermedad estable. Esto se asoció con una disminución en la calcitonina y en el ACE.

El medicamento fue bien tolerado. Se presentó neutropenia grado 4 en 3 pacientes ( $4 \%$ ). Los efectos adversos grado 3 fueron anemia (9\%), hipertensión (16 \%), leucopenia (4\%), diarrea (7\%), neutropenia ( $9 \%$ ) y fatiga, cefalea y aumento en enzimas hepáticas en $1 \%$ de los casos. A la fecha, los pacientes de las presentaciones orales del estudio llevaban más de 15 meses de tratamiento y el $100 \%$ de estos con respuesta continuaban con el medicamento. Los datos de BLU-667 pueden examinarse en detalle en el sitio web de Blueprint Medicines (https://www.blueprintmedicines.com/pipeline/investigational-medicines/)

\section{LOX0 292}

LOXO 292 es una molécula pequeña que inhibe el RET de manera competitiva con el trifosfato de adenosina (ATP). Es altamente selectivo y potente; y puede inhibir mutaciones que predicen resistencia a otros inhibidores multiquinasa. Es activo contra modelos de células humanas con alteraciones en el RET, lo mismo que contra modelos de xenotrasplantes (xenografts) y modelos ortotópicos derivados de tejidos de pacientes (PDX, por sus siglas en inglés: patient-derived xenografts).

El estudio LIBRETO-001 -con datos presentados en Houston (Texas) por la doctora Lori J. Wirth en el MEN 2019: $16^{\text {th }}$ International Workshop on Multiple Endocrine Neoplasia A Focus on New Concepts and Treatment of Malignant Manifestations- es un estudio fase 1 de escalamiento de dosis y evaluación de la farmacocinética. En este se reclutaron 82 pacientes y se evaluó una dosis de $20 \mathrm{mg}$ cada día hasta una dosis de 240 mg cada 12 horas.

Este estudio permitió la inclusión de pacientes con metástasis cerebral ( $n=8,21 \%)$. De acuerdo con los resultados, todos los pacientes con alteraciones en el RET tuvieron al menos estabilidad de la enfermedad. Un paciente tuvo una respuesta completa. Los 4 pacientes que no respondieron no tenían alteraciones en el RET. No hubo ningún efecto adverso grado 4 . Se encontró diarrea grado 3 en el $1 \%$ y cefalea grado 3 en el $1 \%$, las cuales fueron reversibles al suspender la dosis del tratamiento. Los eventos adversos más comunes fueron diarrea y fatiga.

La repuesta fue relativamente rápida, incluso un paciente tuvo síndrome de lisis tumoral. Muchos de estos pacientes habían recibido previamente inhibidores de tirosina quinasa. En este estudio también se incluyeron 29 pacientes con CMT. La mutación más común fue M918T. El resultado en estos pacientes fue muy interesante, ya que se encontró una respuesta parcial en $59 \%$, respuesta completa en $2 \%$ y enfermedad es- 
table en $15 \%$. Adicionalmente, la respuesta fue relativamente rápida en los primeros meses, lo cual estuvo asociado con una caída importante en la calcitonina y el ACE.

También se evaluó el cfDNA en 74 pacientes con alteraciones en el RET. En el 31 \% no se encontró alteración alguna en el RET circulante (DNA). Luego de 2 semanas se documentó una caída importante en el cfDNA. El estudio incluyó, además, un paciente con cáncer de tiroides anaplásico con una fusión en el RET (CCDC6-RET), quien había sido manejado previamente con tiroidectomía, vaciamiento bilateral de cuello, yodo radioactivo, radiocirugía y docetaxel/doxorubicina. El paciente tenía metástasis en el pulmón y en el sistema nervioso central. Este paciente inició LOXO-292 160 mg cada 12 horas y se evidenció una respuesta parcial en el ciclo 3, con una reducción máxima tumoral del 44,8 \%, la cual fue sostenida al cuarto mes. Los datos de LOXO se pueden examinar en detalle en el sitio web de Loxo Oncology (https://www.loxooncology. com/pipeline/ret-program).

\section{Conclusiones}

Los pacientes con cáncer medular de tiroides avanzado tienen pocas opciones terapéuticas. A la fecha, las únicas terapias aceptadas son los inhibidores de tirosina quinasa vandetanib y cabozantinib. Sin embargo, al ser inhibidores multiquinasa tienen efectos adversos importantes que pueden limitar su uso clínico. La inhibición específica del RET por los medicamentos LOXO-292 y BLU-677 parece ser prometedora, dado que tienen una buena respuesta clínica y además tienen efectos adversos menos frecuentes y graves que los inhibidores de tirosina quinasa actuales.

\section{Financiación}

Parte del trabajo fue financiado por la Asociación Colombiana de Endocrinología, Diabetes y Metabolismo.

\section{Conflictos de interés}

Los autores declaran no tener ningún conflicto de interés.

\section{Referencias}

1. Rojas L. Cáncer medular de tiroides. En: Builes C, editor. Tratado de Tiroides. Bogotá: Asociación Colombiana De Endocrinología, Diabetes y Metabolismo; 2014. p. 400-10.

2. Hu MI, Ying AK, Jimenez C. Update on medullary thyroid cancer. Endocrinol Metab Clin North Am. 2014;43(2):423-42.

3. Cabanillas ME, McFadden DG, Durante C. Thyroid cancer. Lancet Lond Engl 2016;388(10061):2783-95.

4. Chala AI, Franco HI, Aguilar CD, Cardona JP. Estudio descriptivo de doce años de cáncer de tiroides, Manizales, Colombia. Rev Colomb Cir. 2010;25(4):276-89.

5. Uricoechea HV, Chaparro JH, Cabrera IM, Delgado VA. Cáncer de Tiroides e Indicaciones de Tiroidectomia. Medicina (Mex). 2015;37(2):109-21.

6. Sánchez G, Gutiérrez C, Valenzuela Á, Tovar JR. Carcinoma diferenciado de tiroides. Rev Colomb Cir. 2014;29:102-9.

7. Castillo JCR, Niño DC, Sánchez EAW, García ME, Camacho PA, Roa JA, et al. Descripción de los hallazgos histopatológicos en la terapia inicial del cáncer de tiroides en un centro de referencia en Bucaramanga, Colombia. Rev Colomb Endocrinol Diabetes Metab. 2017;2(1):40-3.

8. Wandurraga E, Marín L, Natera A, Gómez C, Niño F, Arenas H, et al. Características clínicas, histopatológicas y terapéuticas del cáncer de tiroides en Colombia: serie de 1.096 pacientes. Rev Colomb Endocrinol Diabetes Metab. 2019;6(1):5-12.

9. Hadoux J, Pacini F, Tuttle RM, Schlumberger M. Management of advanced medullary thyroid cancer. Lancet Diabetes Endocrinol. 2016;4(1):64-71.

10. Milling RV, Grimm D, Krüger M, Grosse J, Kopp S, Bauer J, et al. Pazopanib, Cabozantinib, and Vandetanib in the Treatment of Progressive Medullary Thyroid Cancer with a Special Focus on the Adverse Effects on Hypertension. Int J Mol Sci. 2018;19(10).

11. Jhiang SM. The RET proto-oncogene in human cancers. Oncogene. 2000;19(49):5590-7.

12. Takahashi M, Ritz J, Cooper GM. Activation of a novel human transforming gene, ret, by DNA rearrangement. Cell. septiembre de 1985;42(2):581-8.

13. Sabari JK, Siau ED, Drilon A. Targeting RET-rearranged lung cancers with multikinase inhibitors. Oncoscience. 2017;4(3-4):23-4.

14. Waguespack SG, Rich TA, Perrier ND, Jimenez C, Cote GJ. Management of medullary thyroid carcinoma and MEN2 syndromes in childhood. Nat Rev Endocrinol. 2011;7(10):596-607.

15. Wells SA, Asa SL, Dralle H, Elisei R, Evans DB, Gagel RF, et al. Revised American Thyroid Association guidelines for the management of medullary thyroid carcinoma. Thyroid Off J Am Thyroid Assoc. 2015;25(6):567-610.

16. Drilon A, Hu ZI, Lai GGY, Tan DSW. Targeting RET-driven cancers: lessons from evolving preclinical and clinical landscapes. Nat Rev Clin Oncol. 2018;15(3):151-67.
17. BLU-667 Targets RET-Altered Cancers. Cancer Discov. 2018;8(6):OF8.

18. LOXO-292 Reins In RET-Driven Tumors. Cancer Discov. 2018;8(8):904-5.

19. Subbiah V, Gainor JF, Rahal R, Brubaker JD, Kim JL, Maynard M, et al. Precision Targeted Therapy with BLU-667 for RET-Driven Cancers. Cancer Discov. 2018;8(7):836-49.

20. Pietrantonio F, Di Nicolantonio F, Schrock AB, Lee J, Morano F, Fuca G, et al RET fusions in a small subset of advanced colorectal cancers at risk of being neglected. Ann Oncol Off J Eur Soc Med Oncol. 2018;29(6):1394-401.

21. Kato S, Subbiah V, Marchlik E, Elkin SK, Carter JL, Kurzrock R. RET Aberrations in Diverse Cancers: Next-Generation Sequencing of 4,871 Patients. Clin Cancer Res Off J Am Assoc Cancer Res. 2017;23(8):1988-97.

22. Román A, Sierra J, Gutiérrez J, Builes C, Jiménez C. Feocromocitoma-Paraganglioma: revisión de tema. Medicina y Laboratorio. 2015;21(5-6):111-30.

23. Markowitz JN, Fancher KM. Cabozantinib: A Multitargeted Oral Tyrosine Kinase Inhibitor. Pharmacotherapy. 2018;38(3):357-69.

24. Krajewska J, Olczyk T, Jarzab B. Cabozantinib for the treatment of progressive metastatic medullary thyroid cancer. Expert Rev Clin Pharmacol. 2016;9(1):69-79.

25. Frampton JE. Vandetanib: in medullary thyroid cancer. Drugs 2012;72(10):1423-36.

26. Leboulleux S, Bastholt L, Krause T, de la Fouchardiere C, Tennvall J, Awada A, et al. Vandetanib in locally advanced or metastatic differentiated thyroid cancer: a randomised, double-blind, phase 2 trial. Lancet Oncol. 2012;13(9):897-905.

27. Thornton K, Kim G, Maher VE, Chattopadhyay S, Tang S, Moon YJ, et al. Vandetanib for the treatment of symptomatic or progressive medullary thyroid cancer in patients with unresectable locally advanced or metastatic disease: U.S. Food and Drug Administration drug approval summary. Clin Cancer Res Off J Am Assoc Cancer Res. 2012;18(14):3722-30.

28. Wells SA, Robinson BG, Gagel RF, Dralle H, Fagin JA, Santoro M, et al. Vandetanib in patients with locally advanced or metastatic medullary thyroid cancer: a randomized, double-blind phase III trial. J Clin Oncol Off J Am Soc Clin Oncol. 2012;30(2):134-41.

29. Commander H, Whiteside G, Perry C. Vandetanib: first global approval Drugs. 2011;71(10):1355-65.

30. Hu MI, Taylor M, Wirth L, Zhu V, Doebele R, Lee DH, et al. Clinical activity of BLU-667, a highly selective RET inhibitor, in advanced RET-altered thyroid cancers: updated results from the phase 1 ARROW study. :15. [Internet]. blueprintmedicines.com. [citado 1 de noviembre de 2019]. Disponible en https://www.blueprintmedicines.com/wp-content/uploads/2019/01/ ATA_BLU667_Oral-Presentation-060ct2018-Final.pdf 